\title{
CONTROLE POR MODOS DESLIZANTES DE SISTEMAS MULTIVARIÁVEIS NÃO-LINEARES INCERTOS APLICADO À SERVOVISÃO ROBÓTICA NÃO-CALIBRADA
}

\author{
Tiago Roux Oliveira* \\ tiagoroux@uerj.br
}

\author{
Alessandro Jacoud Peixoto ${ }^{\dagger}$ \\ jacoud@poli.ufrj.br
}

\author{
Liu $\mathrm{Hsu}^{\ddagger}$ \\ liu@coep.ufrj.br \\ *Departamento de Engenharia Eletrônica e de Telecomunicações \\ Universidade do Estado do Rio de Janeiro - UERJ \\ Rio de Janeiro, RJ, Brasil \\ ${ }^{\dagger}$ Departamento de Engenharia Eletrônica e de Computação \\ Universidade Federal do Rio de Janeiro - UFRJ \\ Rio de Janeiro, RJ, Brasil \\ ‡Programa de Engenharia Elétrica/COPPE \\ Universidade Federal do Rio de Janeiro - UFRJ \\ Rio de Janeiro, RJ, Brasil
}

\begin{abstract}
Sliding Mode Control of Uncertain Multivariable Nonlinear Systems Applied to Uncalibrated Robotics Visual Servoing

An output-feedback sliding mode controller using monitoring functions was recently introduced for linear uncertain single-input-single-output (SISO) systems with unknown control direction. Here, a generalization is developed for multivariable systems with strong nonlinearities. The monitoring scheme is extended to handle the uncertainty of the plant high frequency gain matrix. Our strategy provides global stability properties and exact output tracking. Experimental results with a robotics visual servoing system, using a fixed but uncalibrated camera, illustrate the robustness and practical viability of the proposed scheme.
\end{abstract}

Artigo submetido em 28/08/2010 (Id.: 01190)

Revisado em 19/11/2010

Aceito sob recomendação do Editor Associado Prof. Marco Henrique Terra
KEYWORDS: multivariable nonlinear systems, unknown control direction, output-feedback, sliding mode control, robotics visual servoing, uncalibrated camera.

\section{RESUMO}

Um controlador por modos deslizantes baseado em realimentação de saída utilizando funções de monitoração foi recentemente introduzido para sistemas monovariáveis lineares incertos com direção de controle desconhecida. Aqui, uma generalização é desenvolvida para sistemas multivariáveis fortemente não-lineares. O esquema de monitoração é estendido para lidar com a incerteza da matriz de ganho de alta frequência da planta. Nossa estratégia garante propriedades globais de estabilidade e rastreamento exato de saída. Resultados experimentais com um sistema de servovisão robótica, utilizando uma câmera fixa mas não-calibrada, ilustram a robustez e a viabilidade prática do esquema proposto. 
PALAVRAS-CHAVE: sistemas multivariáveis não-lineares, direção de controle desconhecida, realimentação de saída, controle por modos deslizantes, servovisão robótica, câmera não-calibrada.

\section{INTRODUÇÃO}

O projeto de controladores por realimentação de saída de sistemas SISO incertos sem a hipótese do conhecimento da direção de controle (sinal do ganho de alta frequência) tem sido um problema instigante desde o começo dos anos 80 (Mudgett \& Morse, 1985). Na literatura de controle adaptativo, o então denominado ganho de Nussbaum (Nussbaum, 1983) vem sendo utilizado na tentativa de relaxar essa hipótese, incluindo o caso de sistemas multivariáveis (Wu \& Zhou, 2004; Zhang $\&$ Ge, 2007). Contudo, essa abordagem é discutível do ponto de vista prático devido a grandes transitórios, excessivo esforço de controle e falta de robustez (Mudgett \& Morse, 1985; Fu \& Barmish, 1986).

Mais recentemente, controladores baseados em realimentação de saída e modos deslizantes para o rastreamento de trajetórias em sistemas SISO incertos lineares e nãolineares com direção de controle desconhecida e grau relativo arbitrário foram introduzidos em (Yan et al., 2008) e (Oliveira et al., 2007), respectivamente. Em lugar do ganho de Nussbaum, a direção de controle foi ajustada a partir de funções de monitoração.

Outras soluções elaboradas são encontradas na literatura de controle por modos deslizantes, entretanto elas estão restritas ao caso SISO de primeira ordem ou com grau relativo dois (Bartolini et al., 2003), (Bartolini et al., 2009), ou ainda, são baseadas em realimentação de estados (Drakunov, 1993).

Neste artigo, estende-se a aplicabilidade da função de monitoração para plantas não-lineares multivariáveis (multi-input-multi-output - MIMO) com grau relativo unitário utilizando-se a abordagem do controle por vetor unitário e modos deslizantes. O novo desafio é que o ganho de alta frequência é uma matriz, não simplesmente um escalar. Além disso, diferentemente de trabalhos anteriores (Hsu et al., 2003; Cunha, 2004) e graças a uma formulação utilizando observadores da norma do estado, aqui os termos não-lineares são permitidos serem dependentes do estado, incluindo não-linearidades fortes (por exemplo, polinomiais) (Oliveira et al., 2010) e descasadas com respeito a entrada de controle. A motivação principal de usarmos o vetor unitário (Baida, 1993; Hsu et al., 2002) em vez da função de chaveamento "sgn(.)" é que um menor conhecimento da matriz de ganho de alta frequência (high frequency gain - HFG) $K_{p}$ é re- querido, quando comparado com as restrições impostas em (Edwards \& Spurgeon, 1998) ou a propriedade de $K_{p}$ ser positiva definida em outras abordagens (Tao \& Ioannou, 1989; Chien et al., 1996). De fato, se o controle vetorial unitário é utilizado, a única exigência sobre a matriz de HFG é que $-K_{p}$ seja Hurwitz e essa condição é necessária e suficiente para a atratividade da superfície de deslizamento como provado em (Baida, 1993; Hsu et al., 2002) ${ }^{1}$.

Neste artigo, $K_{p}$ é desconhecida e incerta. Inspirados pelos recentes esquemas de controle com comutação (Hespanha et al., 2003; Freidovich \& Khalil, 2007; Deaecto \& Geromel, 2009) e nos spectrum-unmixing sets referidos em (Martensson, 1991; Ryan, 1993), propõe-se um mecanismo de chaveamento que escolha uma matriz pré-compensadora $S_{q}$ apropriada em um conjunto indexado finito de matrizes $\mathcal{Q}$ através de uma função de monitoração adequada, de tal forma que a lei de controle vetorial unitária pré-multiplicada por $S_{q}$ possa garantir que $-K_{p} S_{q}$ seja Hurwitz para algum $S_{q}, q \in \mathcal{Q}$. A estabilidade assintótica global com respeito a um conjunto compacto e o rastreamento exato são demonstrados.

Uma aplicação no problema de controle por servovisão com incerteza nos parâmetros de calibração da câmera será usada para ilustrar a eficácia do controlador multivariável proposto em situações práticas. Particularmente, a restrição usual no ângulo de orientação da câmera $\psi \in\left(-\frac{\pi}{2}, \frac{\pi}{2}\right)$, presente em (Papanikolopoulos \& Khosla, 1994; Kelly et al., 1999; Zergeroglu et al., 1999; Leite et al., 2004; Zachi, Lizarralde, Hsu \& Leite, 2006), pode ser removida.

\section{NOTAÇÃO E TERMINOLOGIA}

As seguintes notações e conceitos básicos são empregados ao longo do texto. A norma Euclidiana de um vetor $x$ e a correspondente norma induzida de uma matriz $A$ são denotadas por $|x|$ e $|A|$, respectivamente. O símbolo " $s$ " representa tanto a variável de Laplace quanto o operador diferencial " $d / d t$ ", de acordo com o contexto. A saída $y$ de um sistema linear e invariante no tempo com função de transferência $H(s)$ e entrada $u$ é escrita $y=H(s) u$. O termo genérico $\pi(t)$ é dito exponencialmente decrescente se $|\pi(t)| \leq b e^{-a t}, \forall t \geq 0$ e escalares $a, b>0$, com $b$ podendo depender das condições iniciais do sistema. O conceito de estabilidade ISS (Input-to-StateStable), assim como as definições de funções de classe

\footnotetext{
${ }^{1}$ Como mostrado também em (Baida, 1993; Hsu et al., 2002), nenhuma condição necessária e suficiente é conhecida para o controle por estrutura variável utilizando função de chaveamento " $\operatorname{sgn}(\cdot)$ " de dimensão maior do que dois, i.e., $\operatorname{sgn}(x)=$ $\left[\operatorname{sgn}\left(x_{1}\right) \operatorname{sgn}\left(x_{2}\right) \ldots \operatorname{sgn}\left(x_{n}\right)\right]^{T}$ para $x \in \mathbb{R}^{n}$ e $n>2$.
} 
$\mathcal{K}$ e $\mathcal{K}_{\infty}$ encontram-se de acordo com (Khalil, 2002). Adota-se a definição de Filippov para a solução de equações diferenciais descontínuas (Filippov, 1964) e o conceito de controle equivalente estendido (Hsu et al., 2002), válido dentro e fora da superfície de deslizamento.

\section{FORMULAÇÃO DO PROBLEMA}

Este artigo considera o problema de rastreamento global de sistemas não-lineares MIMO transformáveis na forma normal (Khalil, 2002):

$$
\begin{aligned}
& \dot{\eta}=\phi_{0}(\eta, y, t), \\
& \dot{y}=K_{p} u+\phi_{1}(\eta, y, t),
\end{aligned}
$$

onde $u \in \mathbb{R}^{m}$ é a entrada de controle, $y \in \mathbb{R}^{m}$ é a saída mensurável e os estados $\eta \in \mathbb{R}^{n-m}$ do subsistema- $\eta$, referido por "dinâmica interna", não estão disponíveis. As funções incertas $\phi_{0}$ e $\phi_{1}$ são contínuas por partes em $t$ e localmente Lipschitz contínuas nos outros argumentos. Para cada solução de (1)-(2), existe um intervalo máximo de definição dado por $\left[0, t_{M}\right)$, onde $t_{M}$ pode ser finito ou infinito.

Adicionalmente, considera-se que a matriz de HFG $K_{p}$ é desconhecida (diz-se também que a direção de controle da planta é desconhecida) com parâmetros incertos pertencendo a algum conjunto compacto $\Omega_{p}$. Em $\Omega_{p}$, é assumido apenas que:

(H1) (Direção de Controle) (i) $\operatorname{det}\left(K_{p}\right) \neq 0$, (ii) existe uma constante conhecida $c>0$ tal que $\left|K_{p}^{-1}\right| \leq c$ e (ii) existe um conjunto indexado finito $\mathcal{Q}$ de matrizes conhecidas $S_{q} \in \mathbb{R}^{m \times m}$ tal que $-K_{p} S_{q}$ é Hurwitz para algum $q \in \mathcal{Q}$.

De acordo com (H1), foca-se o caso mais simples de grau relativo um deixando-se o caso de grau relativo arbitrário e direção de controle desconhecida para trabalhos futuros. Como mencionado acima, a condição Hurwitz é necessária e suficiente para a atratividade da superfície de deslizamento no controle por vetor unitário e modos deslizantes (Baida, 1993; Hsu et al., 2002).

Essa hipótese relaxa significativamente a condição essencial de positividade e simetria da matriz $K_{p}$ presente em (Tao \& Ioannou, 1989; Chien et al., 1996). A simetria é uma propriedade não-genérica, podendo ser destruída por incertezas arbitrariamente pequenas. Além disso, se $K_{p}$ é positiva definida, então $-K_{p}$ é Hurwitz, entretanto a recíproca não é verdadeira.

Para o caso SISO, (H1) pode ser interpretada por: as primeiras duas condições indicam que o escalar $K_{p} \neq 0$ pode ser positivo ou negativo, isto é, a direção de controle é desconhecida. Além disso, neste caso o conjunto indexado é $\mathcal{Q}=\{0,1\}$ e um escalar $S_{q}\left(S_{0}=-1, S_{1}=1\right)$ é necessário para fazer $-K_{p} S_{q}$ negativo.

Aqui, um mecanismo de chaveamento baseado em uma função de monitoração proverá uma troca cíclica através dos elementos do conjunto indexado finito $\mathcal{Q}$ (Ryan, 1993). A existência de $\mathcal{Q}$ é garantida pela teoria dos spectrum-unmixing sets apresentada em (Martensson, 1991). A função de monitoração é projetada com base em um limitante para a norma do erro de rastreamento, válido apenas se for selecionada a $S_{q}$ correta. Caso contrário, o chaveamento ocorrerá quando a norma do erro de rastreamento violar este limitante superior.

\subsection{Problema de Rastreamento Global}

O objetivo é encontrar uma lei de controle dinâmica $u$, via realimentação de saída e sem o conhecimento da direção de controle da planta, para levar o erro de saída ou de rastreamento

$$
e(t)=y(t)-y_{m}(t)
$$

assintoticamente ou em tempo finito para zero (rastreamento exato), começando-se de qualquer condição inicial da planta/controlador e mantendo-se todos os sinais da malha fechada limitados uniformemente, apesar das incertezas. A trajetória desejada $y_{m}(t)$ é assumida ser gerada pelo seguinte modelo de referência:

$$
\dot{y}_{m}=A_{m} y_{m}+r, \quad A_{m}=-\operatorname{diag}\left\{\gamma_{1}, \ldots, \gamma_{m}\right\},
$$

onde $r, y_{m} \in \mathbb{R}^{m}, \gamma_{i}>0(i=1, \ldots, m)$ e $r(t)$ é assumida contínua por partes e uniformemente limitada.

A fim de atingir o objetivo de controle, nossa estratégia requer um observador da norma (norm observer) (Sontag \& Wang, 1997; Krichman et al., 2001) para o estado $\eta$ da dinâmica interna (1), de acordo com a seguinte definição e hipótese.

Definição 1 Um observador da norma para o subsistema (1) é um sistema dinâmico SISO de primeira ordem da forma (relembrando que $y$ é a saída da planta):

$$
\dot{\bar{\eta}}=-\lambda_{o} \bar{\eta}+\varphi_{o}(y, t)
$$

com entrada $\varphi_{o}(y, t)$ e saída $\bar{\eta}$, tal que o estado $\eta$ de (1) satisfaz

$$
|\eta(t)| \leq|\bar{\eta}(t)|+\bar{k}_{o}(|\bar{\eta}(0)|+|\eta(0)|) e^{-\lambda_{o} t},
$$

$\forall t \in\left[0, t_{M}\right)$, com alguma constante $\bar{k}_{o}>0$ e para cada estado inicial $\eta(0)$ e $\bar{\eta}(0)$, sendo: (i) $\lambda_{o}>0$ uma constante 
e (ii) $\varphi_{o}(y, t)$ uma função não-negativa, contínua em $y$ e contínua por partes em $t$, satisfazendo $\varphi_{o} \leq \Psi_{o}(|y|)+k_{o}$, para algum $\Psi_{o} \in \mathcal{K}$ e alguma constante $k_{o} \geq 0$.

(H2) (Observabilidade da Norma) O subsistema interno (1) admite um observador da norma conhecido (5) com $\varphi_{o}$ e $\lambda_{o}$ também conhecidos.

É sabido que no caso invariante no tempo, se o subsistema interno (1) é ISS com respeito a $y$, então ele admite tal observador da norma e a planta é de fase mínima.

Na Seção 4 (Observadores da Norma), dois casos são apresentados onde (5) pode ser implementado para o subsistema interno variante no tempo. O primeiro deles incorpora uma classe de não-linearidades $\phi_{0}$ onde uma condição de crescimento linear é imposta apenas no estado não-medido $\eta$. No outro caso, adaptado de (Jiang et al., 2004), ilustra um caso onde não-linearidades polinomiais fortes em $\eta$ são também permitidas. Em ambos os casos, (1) possui uma relação ISS com respeito a funções apropriadas de $y$ e $t$.

De forma a obter um limitante em norma para $\phi_{1} \mathrm{em}$ (2), assume-se que:

(H3) (Funções Limitantes) Existe uma função nãonegativa $\varphi_{1}(|\eta|, y, t)$ conhecida, classe $\mathcal{K}_{\infty}$ e localmente Lipschitz em $|\eta|$, contínua em $y$ e contínua por partes em $t$ tal que $\left|\phi_{1}(\eta, y, t)\right| \leq \varphi_{1}(|\eta|, y, t)$.

Note que, (H3) não é restritiva visto que $\phi_{1}$ é assumida contínua em $\eta$. Além disso, diferentemente de (Hsu et al., 2003; Cunha, 2004), nenhuma condição particular de crescimento é imposta na função limitante $\varphi_{1}$.

\subsection{Do Problema de Rastreamento para o Problema de Regulação}

A partir de (2)-(4), a dinâmica do erro $e$ pode ser escrita como

$$
\dot{e}=A_{m} e+K_{p}\left(u-u^{*}\right),
$$

onde

$$
u^{*}:=K_{p}^{-1}\left(-\phi_{1}+A_{m} y+r\right) .
$$

Deste modo, o problema de rastreamento global pode ser reformulado como um problema de regulação descrito a seguir. Encontrar uma lei de controle $u$ baseada em modos deslizantes e realimentação de saída de forma que, para toda a condição inicial $(\eta(0), e(0), \bar{\eta}(0))$ : (i) as soluções de (2), (5) e (7) são limitadas e (ii) $e(t)$ tende assintoticamente ou em tempo finito para zero.
A lei de controle ideal $u^{*}$ (8) é considerada como uma perturbação de entrada casada em (7). De (H1)-(H3), $u^{*}$ pode ser limitado em norma por sinais disponíveis

$$
\left|u^{*}\right| \leq c\left[\varphi_{1}(2|\bar{\eta}|, y, t)+\left|A_{m} y+r\right|\right]+\pi_{1},
$$

a menos do termo exponencial decrescente $\pi_{1}:=$ $k_{1}(|\bar{\eta}(0)|+|\eta(0)|) e^{-\lambda_{o} t}$, onde $k_{1}>0$ é uma constante, $c$ é dada em (H1) e $\pi_{1}$ vem do termo exponencial em (6). Para obtermos esta desigualdade, utilizamos o fato de que $\varphi_{1}$ é localmente Lipschitz em seu primeiro argumento e que $\chi(a+b) \leq \chi(2 a)+\chi(2 b), \forall a, b \geq 0$ e $\chi \in \mathcal{K}_{\infty}$.

\section{OBSERVADORES DA NORMA}

Neste artigo, assume-se que é possível obter um observador da norma da forma (5) para o estado $\eta$ do sistema interno $\dot{\eta}=\phi_{0}(\eta, y, t)$, dado em (1). Nesta seção, caracterizam-se classes de plantas não-lineares MIMO e exemplos para os quais esse observador da norma possa ser implementado. Em ambos os casos, obtémse uma função de armazenamento $V(\eta)$ satisfazendo $\underline{\alpha}(|\eta|) \leq V(\eta) \leq \bar{\alpha}(|\eta|), \operatorname{com} \underline{\alpha}(\sigma)=\underline{\lambda} \sigma^{2}, \bar{\alpha}(\sigma)=\bar{\lambda} \sigma^{2} \mathrm{e}$ $\underline{\lambda}, \bar{\lambda}$ conhecidos tais que

$$
\frac{\partial V}{\partial \eta} \phi_{0}(\eta, y, t) \leq-\alpha(|\eta|)+\gamma(y, t), \quad \forall t \in\left[0, t_{M}\right),
$$

onde $\alpha \in \mathcal{K}$ e $\gamma$ são funções conhecidas. Além disso, $\gamma$ é uma função não-negativa, contínua em $y$ e contínua por partes em $t$ satisfazendo $\varphi \leq \Psi_{\gamma}(|y|)+k_{\gamma}$, para algum $\Psi_{\gamma} \in \mathcal{K}$ e constante $k_{\gamma} \geq 0$. Isso assegura que o sistema interno (1) tem uma relação ISS de $\gamma$ para $\eta$, o que corresponde a uma generalização do conceito de fase mínima e permite concluir que se $y$ estiver limitada, $\eta$ também estará.

A função classe- $\mathcal{K} \alpha_{1}:=\alpha \circ \bar{\alpha}^{-1}$ é dita ultimately linearly lower bounded (ULLB), significando que existe $\epsilon \geq 0$, tal que $\alpha_{1}(\sigma) \geq 2 \lambda \sigma, \forall \sigma \geq \epsilon$ e algum $\lambda>0$. Em particular, se $\alpha_{1}$ é linear, i.e., se $\alpha_{1}(\sigma)=2 \lambda_{1} \sigma$ (para algum $\lambda_{1}>0$ ), podemos fazer $\epsilon=0$ e $\lambda \leq \lambda_{1}$. Além disso, se $\alpha_{1}$ é nãolinear, podemos tomar $\epsilon>0$ e $\lambda<\alpha_{1}(\epsilon) /(2 \epsilon)$. Note que a propriedade ULLB é menos conservativa que o conceito de função stiffening ${ }^{2}$ introduzido em (Arcak et al., 2002) no contexto de estabilidade absoluta.

Levando em consideração a função ULLB $\alpha_{1}$ detalhada acima, o observador da norma (5) pode enfim ser obtido seguindo os próximos passos.

\footnotetext{
${ }^{2}$ De acordo com (Arcak et al., 2002), a não-linearidade $\alpha_{1}(\sigma)$ é stiffening se para todo $\lambda>0$, existe $\epsilon>0$ tal que $\sigma \geq \epsilon \Rightarrow$ $\alpha_{1}(\sigma) \geq \lambda \sigma$. Em outras palavras, $\alpha_{1}(\sigma) / \sigma$ é crescente e nãonegativa no intervalo $\sigma \in(0, \infty)$ e portanto $\alpha_{1}(\cdot)$ cresce mais rapidamente que uma função linear.
} 
Considere a notação $\dot{V}=\frac{\partial V}{\partial \eta} \phi_{0}(\eta, y, t)$ e escolha $\lambda$ como descrito acima para um dado $\epsilon \geq 0$. A partir de (10), pode-se escrever $\dot{V} \leq-\alpha_{1}(V)+\gamma(y, t)$ ou, equivalentemente,

$$
\dot{V} \leq-2 \lambda V+\left[2 \lambda V-\alpha_{1}(V)\right]+\gamma(y, t) .
$$

Agora, dado um $V$, tem-se que $V \leq \epsilon$ ou $V>\epsilon$. Portanto, $\dot{V} \leq-2 \lambda V+\left[2 \lambda \epsilon+\alpha_{1}(\epsilon)\right]+\gamma$ ou $\dot{V} \leq-2 \lambda V+\gamma$, e consequentemente $\dot{V} \leq-2 \lambda V+\left[2 \lambda \epsilon+\alpha_{1}(\epsilon)\right]+\gamma$. Assim sendo, utilizando-se o Teorema da Comparação (Khalil, 2002), conclui-se que

$$
V \leq e^{-2 \lambda t} * \gamma_{1}(y(t), t)+V(\eta(0)) e^{-2 \lambda t},
$$

onde $\gamma_{1}=\gamma+2 \lambda \epsilon+\alpha_{1}(\epsilon)$ é conhecido e $*$ denota o operador convolução. Finalmente, pode-se implementar um observador da norma da forma (5) para o estado $\eta, \operatorname{com} \varphi_{o}(y, t)=\sqrt{\gamma_{1}(y, t) / \underline{\lambda}}$ e $\lambda_{o}=\lambda$, aplicando-se a função $\underline{\alpha}^{-1}$ em ambos os lados da última desigualdade.

\subsection{Sistema interno com crescimento linear no estado não-medido}

Considere a classe de plantas não lineares MIMO (1)-(2) com a função $\phi_{0}$ dada por

$$
\phi_{0}(\eta, y, t)=A_{0} \eta+\bar{\phi}_{0}(\eta, y, t),
$$

onde $A_{0}$ e $\bar{\phi}_{0}$ podem ser incertas. Considera-se que todas as incertezas paramétricas pertençam a um conjunto compacto $\Omega_{0}$ tal que os limitantes para as incertezas necessários para o projeto do observador da norma estejam disponíveis.

Em $\Omega_{0}$, assume-se que: (i) $A_{0}$ é Hurwitz, (ii) existem constantes positivas conhecidas $c_{0}, c_{1}, c_{2}$ tal que $|P|<c_{0}$, $\lambda_{\max }(P)<c_{1}$ e $\lambda_{\min }(P)>c_{2}$, onde $P=P^{T}>0$ é a solução de $A_{0}^{T} P+P A_{0}=-I$ e (iii) existe uma constante conhecida $\mu \geq 0$ e uma função também conhecida $\varphi_{0}$ tal que

$$
\left|\bar{\phi}_{0}\right| \leq \mu|\eta|+\bar{\varphi}_{0}(y, t),
$$

onde $\bar{\varphi}_{0}$ é não-negativa, contínua em $y$, contínua por partes em $t$ e satisfaz $\bar{\varphi}_{0} \leq \Psi_{0}(|y|)+k_{0}$, para algum $\Psi_{0} \in \mathcal{K}$ e alguma constante $k_{0} \geq 0$. Agora, utilizandose a seguinte função quadrática $V(\eta)=\eta^{T} P \eta$ pode-se obter a desigualdade (10) com

$$
\alpha(|\eta|)=\frac{|\eta|^{2}}{2} \quad \text { e } \quad \gamma(y, t)=\frac{8 c_{0}^{2}}{1-2 c_{0} \mu} \bar{\varphi}_{0}^{2}(y, t),
$$

contanto que $\mu<1 /\left(2 c_{0}\right)$. Portanto, a função $\alpha_{1}(\sigma)=$ $\sigma /\left(2 \lambda_{\max }(P)\right)$ e definindo $c_{3}=8 c_{0}^{2} /\left(1-2 c_{0} \mu\right)$, o $o b$ servador da norma (5) pode ser implementado com $\lambda_{o}<1 /\left(4 c_{1}\right)$ e $\varphi_{o}(y, t)=\sqrt{c_{3} / c_{2}} \bar{\varphi}_{0}(y, t)$.

\subsection{Sistema interno sem restrição linear de crescimento no estado não-medido}

Para ilustrar que a aplicabilidade da estratégia proposta não está restrita a plantas não-lineares com $\phi_{0}(\eta, y, t)$ limitado de forma afim com a norma do estado nãomedido $\eta$, como em (11) e (12), considera-se um caso simples invariante no tempo adaptado de (Jiang et al., 2004 ), onde $\phi_{0} \in \mathbb{R}$ é dado por

$$
\phi_{0}(\eta, y, t)=-\eta^{5}-\eta^{2}|y|^{2} .
$$

Neste caso, a dinâmica do estado $\eta$ é ISS com respeito a $y$ e a função de Lyapunov-ISS (Jiang et al., 2004) é $V(\eta)=\eta^{2} / 2$. Assim, tem-se que $\dot{V}=-\eta^{6}-\eta^{3}|y|^{2}$ e aplicando-se a desigualdade de Young ao termo $\eta^{3}|y|^{2}$ tem-se que $\eta^{3}|y|^{2} \leq \eta^{6} / 2+|y|^{4} / 2$ e, consequentemente, $\dot{V} \leq-\eta^{6} / 2+|y|^{4} / 2$. Por esta razão, pode-se obter a desigualdade (10) com $\alpha(|\eta|)=\eta^{6} / 2$ e $\gamma(y, t)=|y|^{4} / 2$. Assim sendo, $\alpha_{1}(\sigma)=\sigma^{3} / 2$ e o observador da norma (5) pode ser implementado com $\lambda_{o}<\epsilon^{2} / 4$ e $\varphi_{o}(y, t)=$ $\sqrt{|y|^{4} / 2+2 \lambda_{o} \epsilon+\epsilon^{3} / 2}$.

\section{CONTROLE VETORIAL UNITÁRIO VIA REALIMENTAÇÃO DE SAÍDA}

Essa seção e a próxima apresentam a primeira generalização dos controladores propostos em (Yan et al., 2008) e (Oliveira et al., 2007) para uma classe de plantas nãolineares MIMO com direção de controle desconhecida e grau relativo unitário.

Seja $q^{*}$ o índice desconhecido do conjunto indexado finito $\mathcal{Q}$ dado em (H1) para o qual a matriz correspondente $\mathcal{S}=S_{q^{*}}$ também desconhecida assegura que $-K_{p} \mathcal{S}$ seja Hurwitz. Portanto, a equação de Lyapunov $\left(K_{p} \mathcal{S}\right)^{T} P+$ $P\left(K_{p} \mathcal{S}\right)=I$ tem uma única solução $P=P^{T}>0$.

Agora, se a direção de controle fosse conhecida ( $q^{*}$ conhecido), poderíamos aplicar a seguinte lei de controle vetorial unitário ${ }^{3}$ (UVC) (Hsu et al., 2002)

$$
u=-\mathcal{S} \varrho(\bar{\eta}, y, t) \frac{e}{|e|}, \quad e \neq 0,
$$

em (7) e verificar que, se a função de modulação $\varrho$ satisfaz

$$
\varrho \geq c_{d}\left|u^{*}(t)\right|+c_{e}|e|+\delta, \quad \delta \geq 0,
$$

a menos do termo exponencial decrescente $c_{d} \pi_{1}$, então a derivada de Dini temporal de $V=\sqrt{e^{T} P e}$ ao longo das

\footnotetext{
${ }^{3}$ Visto que $e=0$ corresponde a um conjunto de medida nula no sentido de Lebesgue, então, de acordo com a Teoria de Filippov, o valor do sinal de controle neste ponto é irrelevante. Contudo, com a finalidade de manter a consistência matemática, assume-se que $u=0$ se $e=0$.
} 
soluções de (7) satisfaz:

$$
\dot{V} \leq-\lambda_{m} V+\frac{c_{d} \pi_{1}}{2 \sqrt{\lambda_{\min }(P)}}, \quad \forall t \in\left[t_{i}, t_{M}\right),
$$

para qualquer $t_{i} \in\left[0, t_{M}\right)$, onde $0<\lambda_{m}<\min _{i}\left\{\left|\gamma_{i}\right|\right\}, i=$ $1, \ldots, m$ em (4), $c_{d} \geq 2\left|P K_{p}\right|, c_{e} \geq\left|A_{m}^{T} P+P A_{m}\right|+\lambda_{m}$ e $\pi_{1}$ vindo de (9). Além disso, se $\gamma_{i}=\lambda_{m}(\forall i)$, podese escolher $c_{e}=0$. A partir do Lema da Comparação (Filippov, 1964), tem-se que:

$|e(t)| \leq \zeta(t), \zeta(t):=\left|e\left(t_{i}\right)\right| e^{-\lambda_{m}\left(t-t_{i}\right)}+\pi_{2}, \forall t \in\left[t_{i}, t_{M}\right)$,

$\operatorname{com} \pi_{2}:=\Psi_{2}(|\bar{\eta}(0)|+|\eta(0)|) e^{-\lambda_{c} t}, \Psi_{2} \in \mathcal{K}$ e $0<\lambda_{c}<$ $\min \left\{\lambda_{o}, \lambda_{m}\right\}$ (vide Hsu et al. (2002, Lemma 1) para maiores detalhes).

O principal problema é que $q^{*}$ é desconhecido e, portanto, não podemos implementar a lei UVC em (14). Em (Yan et al., 2008) e (Oliveira et al., 2007), um esquema de chaveamento baseado em função de monitoração foi desenvolvido para lidar com a falta de conhecimento da direção de controle. Entretanto, apenas plantas SISO foram consideradas. Naquele caso, $K_{p}$ era um escalar e depois de um número finito de trocas do sinal da lei de controle $\left(S_{q}= \pm 1\right)$, a direção de controle correta pôde ser detectada. Para plantas não-lineares MIMO, a lei UVC é redefinida como

$$
u=-S_{q} \varrho(\bar{\eta}, y, t) \frac{e}{|e|}, \quad e \neq 0, \quad \forall t \in\left[0, t_{M}\right),
$$

onde $\varrho$ satisfaz (15) e o mecanismo de chaveamento também baseado em função de monitoração é utilizado para decidir quando a matriz estática pré-compensadora $S_{q}$ (Hespanha et al., 2003) deva ser chaveada dentro da coleção de matrizes com $q \in \mathcal{Q}$.

\section{ESQUEMA DE CHAVEAMENTO FUNÇÃO DE MONITORAÇÃO}

Agora construiremos a função de monitoração $\varphi_{m}$ baseada em um limitante para a norma de $e$ dado em (16). Relembrando que (16) é válido apenas se a matriz $S_{q}$ é correta $\left(S_{q}=\mathcal{S}\right)$, parece natural utilizarmos $\zeta$ como benchmark e decidir quando um chaveamento de $S_{q}$ é necessário, ou seja, o chaveamento ocorrerá apenas quando (16) for violada. Todavia, como $\pi_{2}$ não é mensurável, considera-se a seguinte função, definida no intervalo $\left[t_{k}, t_{k+1}\right)$, para substituir $\zeta$ :

$$
\varphi_{k}(t)=\left|e\left(t_{k}\right)\right| e^{-\lambda_{m}\left(t-t_{k}\right)}+a(k) e^{-\lambda_{c} t},
$$

onde o instante de chaveamento $t_{k}$ ajusta a mudança de índice $q \in \mathcal{Q}$, e assim comuta ciclicamente as matrizes $S_{q}$, e $a(k)$ é qualquer sequência positiva monotonicamente crescente e ilimitada. A função de monitoração $\varphi_{m}$ pode, portanto, ser definida como

$$
\varphi_{m}(t):=\varphi_{k}(t), \quad \forall t \in\left[t_{k}, t_{k+1}\right) \subset\left[0, t_{M}\right) .
$$

Note que a partir de (18) e (19), tem-se que $\left|e\left(t_{k}\right)\right|<$ $\varphi_{k}\left(t_{k}\right)$ para $t=t_{k}$. Por esta razão, o instante de chaveamento $t_{k}$ é definido quando a função de monitoração $\varphi_{m}(t)$ encontra $|e(t)|$, isto é,

$t_{k+1}:= \begin{cases}\min \left\{t>t_{k}:|e(t)|=\varphi_{k}(t)\right\}, & \text { se este existir, } \\ t_{M}, & \text { caso contrário, }\end{cases}$

onde $k \in\{0,1, \ldots\}$ e $t_{0}:=0$ (vide Figura 1). A seguinte desigualdade é obtida diretamente da definição (19)

$$
|e(t)| \leq \varphi_{m}(t), \quad \forall t \in\left[0, t_{M}\right) .
$$

A Figura 1 ilustra a norma do erro de rastreamento $|e|$ assim como a função de monitoração $\varphi_{m}$.

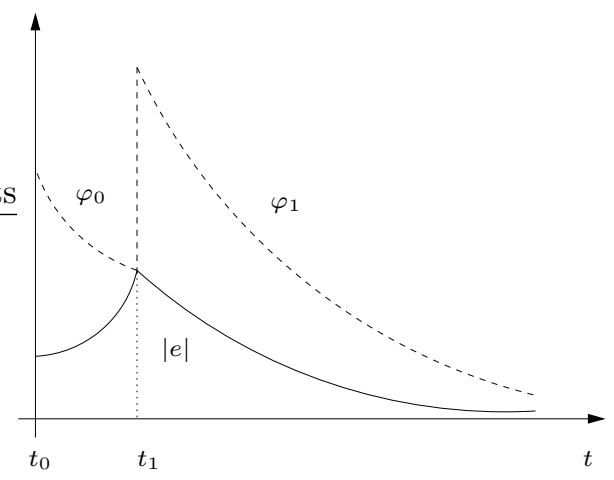

Figura 1: Trajetórias de $\varphi_{m}$ e $|e|$.

\section{Observação 1 (Soluções Ilimitadas e Infinitos} Chaveamentos) De acordo com (H2) e a Definição 1, o sistema em malha fechada (1)-(2) com a lei de controle (17) tem uma propriedade denominada unboundedness observability (UO), no sentido de que qualquer escape em tempo finito (ou mesmo infinito) de algum sinal do sistema pode ser observado na saída $y$. Assim, qualquer eventual escape é evitado se $y$ (ou o erro de rastreamento $e$ ) permanece uniformemente limitado. Portanto, pela "característica exponencial decrescente" da função de monitoração $\varphi_{m}$ em (19), é claro que soluções ilimitadas podem ocorrer apenas se $\varphi_{m}$ apresentar um infinito número de chaveamentos $(k \rightarrow+\infty)$.

\section{ANÁLISE DE ESTABILIDADE}

A fim de levar em conta todas as condições iniciais do sistema em malha fechada, considere

$$
z^{T}(t):=\left[z^{0}(t), e^{T}(t)\right], z^{0}(t):=[|\eta(0)|,|\bar{\eta}(0)|] e^{-\gamma t},
$$


onde $z^{0}$ denota o estado transitório (Hsu et al., 2002) e $\gamma>0$ é uma constante genérica. O resultado principal é agora estabelecido.

Teorema 1 Considere sistemas não-lineares transformáveis na forma normal (1)-(2) com lei de controle UVC (17) e função de monitoração (18)-(19). Assuma que (H1)-(H3) sejam verificadas. Se a função de modulação satisfaz (15), então o chaveamento da direção de controle termina após um número finito de chaveamentos. O sistema completo do erro (7) com estado z(t)é globalmente assintoticamente estável com respeito a um conjunto compacto independente das condições iniciais e por fim exponencialmente convergente para zero. Além disso, todos os sinais do sistema em malha fechada permanecem uniformemente limitados e se $\delta>0$ em (15), então o modo deslizante $e \equiv 0$ é alcançado em tempo finito.

Prova: Ver Apêndice A.

Observação 2 (Conjuntos Compactos e Transitórios Iniciais) Note que os resultados de estabilidade com respeito a um conjunto compacto, não necessariamente pequeno, leva em conta o transitório inicial enquanto a função de monitoração ainda não parou de chavear. Isso significa que, mesmo que os erros iniciais sejam muito pequenos, o transitório inicial pode não ser correspondentemente pequeno.

Observação 3 (Seleção da Matriz $S_{q}$ ) Sabe-se que se $-K_{p} S_{q}$ é Hurwitz todas as trajetórias do sistema convergem para a origem do espaço de estados do erro (Hsu et al., 2002, Lemma 1). Além disso, se $-K_{p} S_{q}$ não é Hurwitz, então para quase toda condição inicial (ou seja, exceto para um conjunto de medida nula) as trajetórias do sistema divergem ilimitadamente ou não convergem para a origem. Isso é uma contradição, visto que se o processo de chaveamento da direção de controle termina, de acordo com o Teorema 1, o estado do erro deve convergir para a origem. Então, quase sempre, a última matriz $S_{q}$ selecionada faz com que $-K_{p} S_{q}$ seja Hurwitz.

\section{Observação 4 (Grau Relativo Arbitrário)}

A generalização para sistemas não-lineares com grau relativo arbitrário é consideravelmente mais complexa. Alguns resultados preliminares podem ser encontrados em (Oliveira et al., 2007) para o caso SISO. Analogamente a (Nunes et al., 2010), a idéia chave para superar o obstáculo do grau relativo é introduzir um compensador híbrido combinando, através de chaveamento, um observador de alto ganho (Khalil, 2002) com um diferenciador robusto e exato, baseado em modos deslizantes de ordem superior (Fridman et al., 2008), para atingirmos estabilidade global prática e uniforme, além de rastreamento assintótico exato.

Observação 5 (Direção de Controle Variante no Tempo) Devido a propriedade de decaimento exponencial da função de monitoração, o esquema aqui desenvolvido pode ser eficaz no tratamento de sistemas com direção de controle variante no tempo, ou seja $K_{p}(t)$. Contudo, é correto pensar que essa dependência com o tempo também não poderá ser arbitrária, o que demanda alguma hipótese sobre o comportamento das mudanças da direção de controle e ao mesmo tempo evitar o fenômeno de escape em tempo finito não excluído a priori para os sistemas não-lineares aqui tratados. Neste sentido, um primeiro caminho seria assumir que as mudanças na direção de controle são tais que estas não possam resultar em um comportamento Zeno ou no fenômeno de Fuller (Van der Schaft \& Schumacher, 2000), isto é, não poderá haver um número infinito de chaveamentos da função de monitoração em um intervalo de tempo finito.

\section{APLICAÇÃO AO PROBLEMA DE SERVOVISÃO}

Para ilustar a aplicabilidade do esquema de chaveamento e monitoração proposto, considera-se um caso simples onde a planta é um integrador MIMO sem subsistema interno. O esquema de controle proposto é utilizado para resolver o problema de controle por servovisão para um manipulador robótico de 6-DOF (degrees of freedom) realizando movimentos planares no espaço cartesiano e utilizando uma câmera fixa, não-calibrada, com eixo óptico ortogonal ao espaço de trabalho do robô.

O modelo simplificado para o movimento de um ponto do efetuador do robô (alvo) no sistema de coordenadas da imagem da câmera, assim como os sistemas de coordenadas do robô, da imagem e da câmera são ilustrados nas Figuras 2 e 3.

\subsection{Controle Cinemático Servovisual}

Primeiramente, considera-se o problema de controle cinemático para um robô manipulador não-redundante. Nesta abordagem, a posição do efetuador do robô $x \in \mathbb{R}^{n}$ é dada pelo mapeamento cinemático direto $x=f(\theta)$, onde $\theta \in \mathbb{R}^{m}$ é o vetor de ângulos das juntas do manipulador e $m \geq n$.

A cinemática diferencial pode ser obtida a partir da derivada temporal do mapeamento cinemático direto sendo dada por

$$
\dot{x}=J(\theta) \dot{\theta},
$$




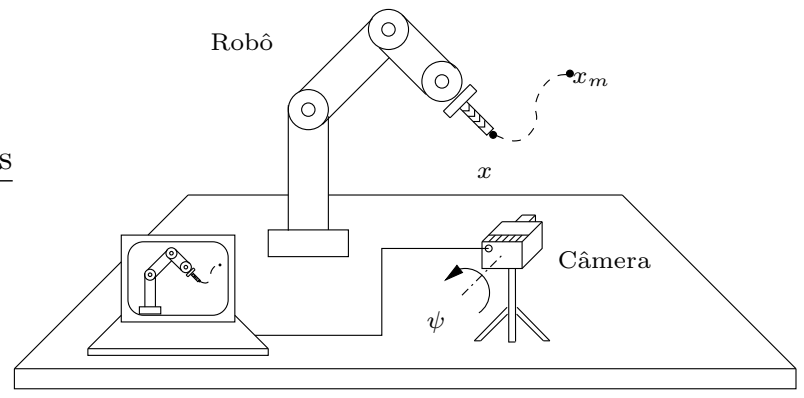

Figura 2: Problema de servovisão: posições do efetuador do robô $x$ e desejada $x_{m}$.

onde $J(\theta)=\frac{\partial f}{\partial \theta} \in \mathbb{R}^{n \times m}$ é o Jacobiano do manipulador. Esse modelo pode ser aplicado a maioria dos robôs comerciais com elevado fator de redução nas engrenagens e/ou quando a velocidade da tarefa é lenta. Assim sendo, considerando $\dot{\theta}_{i}$ como a entrada de controle $v_{i}$ $(i=1, \cdots, m)$, obtém-se o seguinte sistema

$$
\dot{x}=J(\theta) v .
$$

Uma lei de controle cartesiana $u$ pode ser transformada em sinais de controle das juntas utilizando-se

$$
v=J^{-1}(\theta) u,
$$

desde que $u$ não leve o robô manipulador para configurações com singularidades, ou seja, $J(\theta)$ é não-singular.

Agora o problema de controle por servovisão de um robô manipulador será considerado. Neste contexto, a abordagem por servovisão é utilizada para fecharmos a malha de controle de posição do efetuador do robô. Seja $y \in \mathbb{R}^{2}$ a posição do efetuador e $y_{m} \in \mathbb{R}^{2}$ a trajetória desejada a ser efetuada por um alvo fixado na extremidade do braço robótico, ambas expressas no plano de coordenadas da imagem da câmera. O objetivo de controle pode ser descrito por fazer

$$
y \rightarrow y_{m}(t), \quad e=y-y_{m} \rightarrow 0,
$$

onde $e \in \mathbb{R}^{2}$ é o erro de imagem.

Aqui, considera-se que o robô manipulador realiza movimentos planares no espaço cartesiano e, portanto, $n=m=2$ e $x \in \mathbb{R}^{2}$. Considerando uma câmera CCD (charge-coupled device) monocular fixa com eixo óptico perpendicular ao sistema de coordenadas do robô, a transformação câmera/espaço de trabalho (Hutchinson et al., 1996) pode ser representada por

$$
y=K_{p} x+y_{0},
$$

com

$$
K_{p}=\frac{f_{0}}{f_{0}+z_{0}}\left[\begin{array}{cc}
\alpha_{1} & 0 \\
0 & \alpha_{2}
\end{array}\right]\left[\begin{array}{rr}
\cos (\psi) & -\operatorname{sen}(\psi) \\
\operatorname{sen}(\psi) & \cos (\psi)
\end{array}\right],
$$

onde $y_{0}$ é um termo constante que depende da posição do sistema de coordenadas da câmera com respeito ao sistema de coordenadas do robô, $K_{p}$ é a matriz de transformação câmera/espaço de trabalho e considera o ângulo de orientação ou desalinhamento da câmera $\psi$ (vide Figura 2) com respeito ao sistema de coordenadas do robô, $f_{0}$ é a distância focal da câmera, $z_{0}$ é a distância (profundidade) do sistema de coordenadas da imagem da câmera com relação ao espaço de trabalho do robô (em geral $z_{0} \gg f_{0}$ ), e $\alpha_{1}, \alpha_{2}>0$ são fatores de escala da câmera $[\mathrm{pixel} / \mathrm{mm}]$.

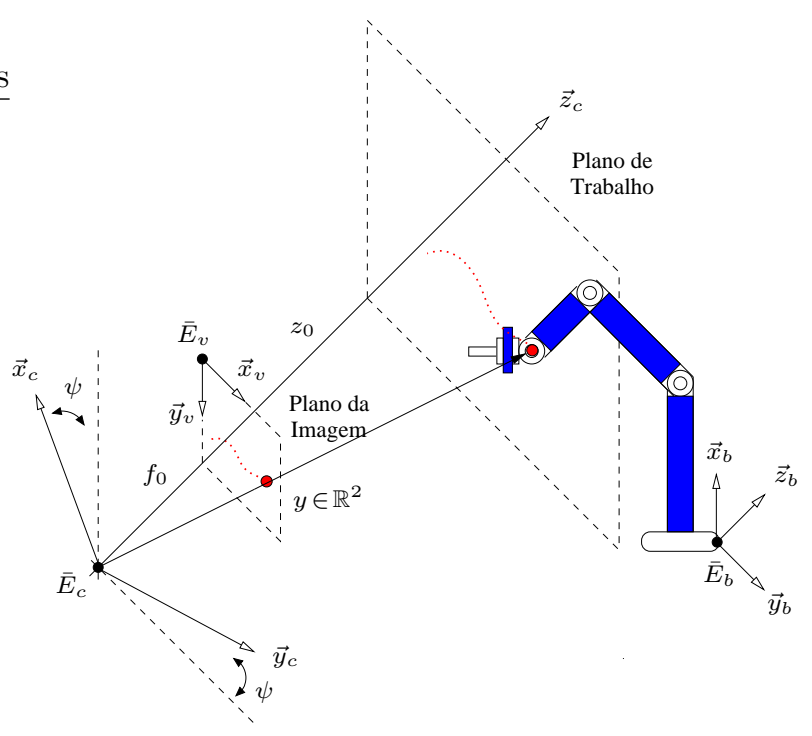

Figura 3: Sistemas de Coordenadas: câmera $\bar{E}_{c}=\left[\begin{array}{lll}\vec{x}_{c} & \vec{y}_{c} & \vec{z}_{c}\end{array}\right]$, imagem $\bar{E}_{v}=\left[\begin{array}{lll}\vec{x}_{v} & \vec{y}_{v} & \vec{z}_{v}\end{array}\right]$ e robô $\bar{E}_{b}=\left[\begin{array}{lll}\vec{x}_{b} & \vec{y}_{b} & \vec{z}_{b}\end{array}\right]$. Note que $\bar{E}_{c}$ e $\bar{E}_{b}$ estão alinhados apenas com respeito ao eixo- $z$.

O problema de controle cartesiano no sistema de coordenadas da imagem da câmera é descrito a partir de (26) por

$$
\dot{y}=K_{p} u,
$$

onde $u \in \mathbb{R}^{2}$ é a lei de controle a ser projetada.

Baseado em (27) e considerando uma lei de controle feedforward mais proporcional dada por

$$
u=K_{p}^{-1}\left[\dot{y}_{m}+K\left(y_{m}-y\right)\right],
$$

tem-se que a dinâmica do erro de imagem é governada por $\dot{e}+K e=0$. Assim, por uma escolha apropriada da matriz positiva definida $K, e \rightarrow 0$ exponencialmente quando $t \rightarrow+\infty$. 
Entretanto, assumindo que os parâmetros intrínsecos e extrínsecos do modelo da câmera são incertos (câmera não-calibrada), a matriz de transformação $K_{p}$ também é incerta. Consequentemente, a lei de controle (28) não garante rastreamento assintótico da trajetória desejada, uma vez que o sistema em malha fechada não é perfeitamente linearizado por realimentação.

Neste contexto, alguns esquemas adaptativos foram propostos com o intuito de lidar com as incertezas nos parâmetros de calibração da câmera (Papanikolopoulos \& Khosla, 1994; Kelly et al., 1999; Zergeroglu et al., 1999; Astolfi et al., 2002; Zachi, Hsu, Ortega \& Lizarralde, 2006). Contudo, é sabido que estratégias adaptativas podem resultar em comportamento transitório ruim e falta de robustez a dinâmicas não-modeladas. Além disso, devido a restrições dos algoritmos de controle, nessas abordagens o ângulo de orientação da câmera $\psi$ deve ser escolhido dentro da faixa $\left(-\frac{\pi}{2}, \frac{\pi}{2}\right)$.

\subsection{Controle Vetorial Unitário para Servovisão}

No que se segue, a combinação do controlador vetorial unitário e o esquema de chaveamento baseado em função de monitoração é aplicada para resolver o problema de controle por servovisão de manipuladores robóticos na presença de incertezas nos parâmetros da câmera e sujeito a qualquer desalinhamento do ângulo da mesma.

Para ilustrar a propriedade de rejeição de perturbação do esquema proposto, adicionamos uma perturbação artificial $d(\theta)=\left[\begin{array}{ll}\theta_{1}^{2} & \theta_{2}^{2}\end{array}\right]^{T}$ à entrada de controle $u$ utilizando-se medidas $\theta$ obtidas de encoders do robô. Então, a partir do mapeamento cinemático inverso e (26), o problema de controle cartesiano no sistema de coordenada da imagem pode ser reescrito como $(2)$ com um $\phi_{1}(y, t)$ apropriado.

A função de monitoração $\varphi_{m}$ (18)-(19) é utilizada para trocar a matriz $S_{q}$ em (17). O conjunto finito de matrizes $S_{q}, q \in \mathcal{Q}=\{0,1,2,3\}$ pode ser escolhido como:

$S_{0}=\left[\begin{array}{rr}0 & 1 \\ -1 & 0\end{array}\right], S_{1}=\left[\begin{array}{ll}1 & 0 \\ 0 & 1\end{array}\right], S_{2}=\left[\begin{array}{rr}0 & -1 \\ 1 & 0\end{array}\right], S_{3}=\left[\begin{array}{rr}-1 & 0 \\ 0 & -1\end{array}\right]$.

Assim, para qualquer ângulo de desalinhamento $\psi$, $-K_{p} S_{q}$ é Hurwitz para algum $S_{q}$ e a restrição usual $|\psi|<\frac{\pi}{2}$ pode ser removida. O diagrama em blocos do sistema de controle é apresentado na Figura 4.

\section{EXPERIMENTOS E RESULTADOS}

Nesta seção descreve-se o ambiente experimental utilizado para a obtenção dos resultados e testes realizados que ilustram o desempenho do esquema de controle.

\subsection{Ambiente Experimental}

Os resultados experimentais foram obtidos através da implementação do controlador proposto em um robô manipulador de 6-DOF Zebra Zero (Integrated Motions, Inc.). Os efeitos dinâmicos são desprezíveis neste robô devido ao seu elevado fator de redução nas engrenagens das juntas e ao alto ganho na sua malha de controle de velocidade.

Uma câmera KP-D50 CCD (Hitachi, Ltd.) com distância focal $f_{0}=6[\mathrm{~mm}]$ e fatores de escala $\alpha_{1}=119$ e $\alpha_{2}=102[\mathrm{pixel} / \mathrm{mm}]$ foi montada em frente ao Zebra Zero (vide Figura 5 do ponto de vista da câmera $\operatorname{com} \psi \approx 0$ ). A profundidade média do plano da imagem para o espaço de trabalho do robô é $z_{0}=1[\mathrm{~m}]$. A característica da imagem (target) extraída são as coordenadas do centróide de um disco vermelho fixado no punho do robô. As imagens de 640×480 [pixel] são capturadas utilizandose uma placa Meteor frame-grabber (Matrox, Ltd.) com taxa de amostragem de 30 frames por segundo.

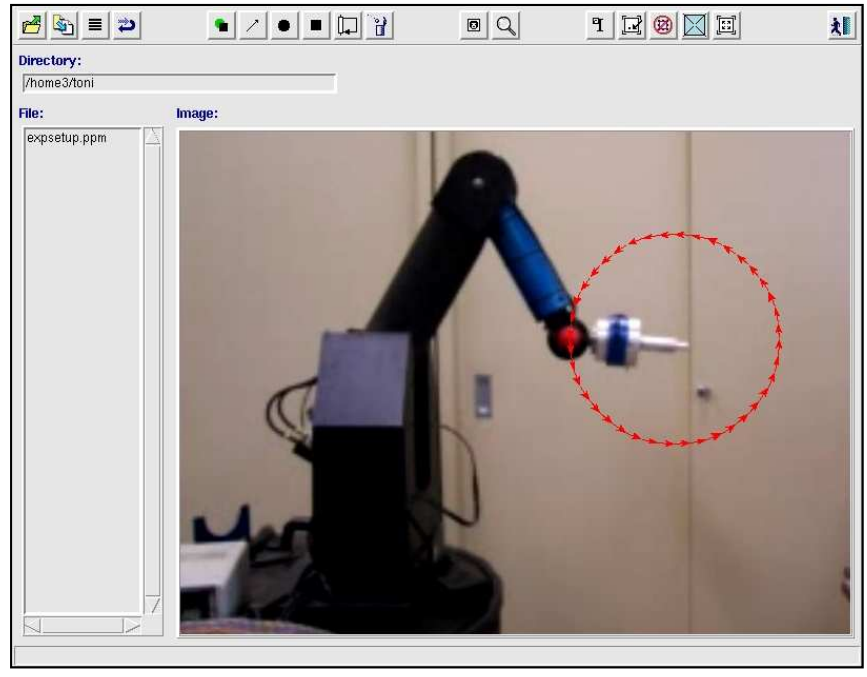

Figura 5: Ambiente Experimental.

O controlador servovisual é codificado em linguagem $\mathrm{C}$ e executado a cada 35.0 [ms] em um processador Pentium Pro $200 \mathrm{MHz}$ com 64 Mbytes de memória RAM utilizando o sistema operacional Linux. O controle de velocidade das juntas gerado pela lei de controle servovisual alimenta a placa ISA do Zebra Zero, fechando a malha de velocidade a partir de um microcontrolador HCTL1100 (HP Inc.) operando em modo de velocidade proporcional com período de amostragem de 0.52 [ms]. 


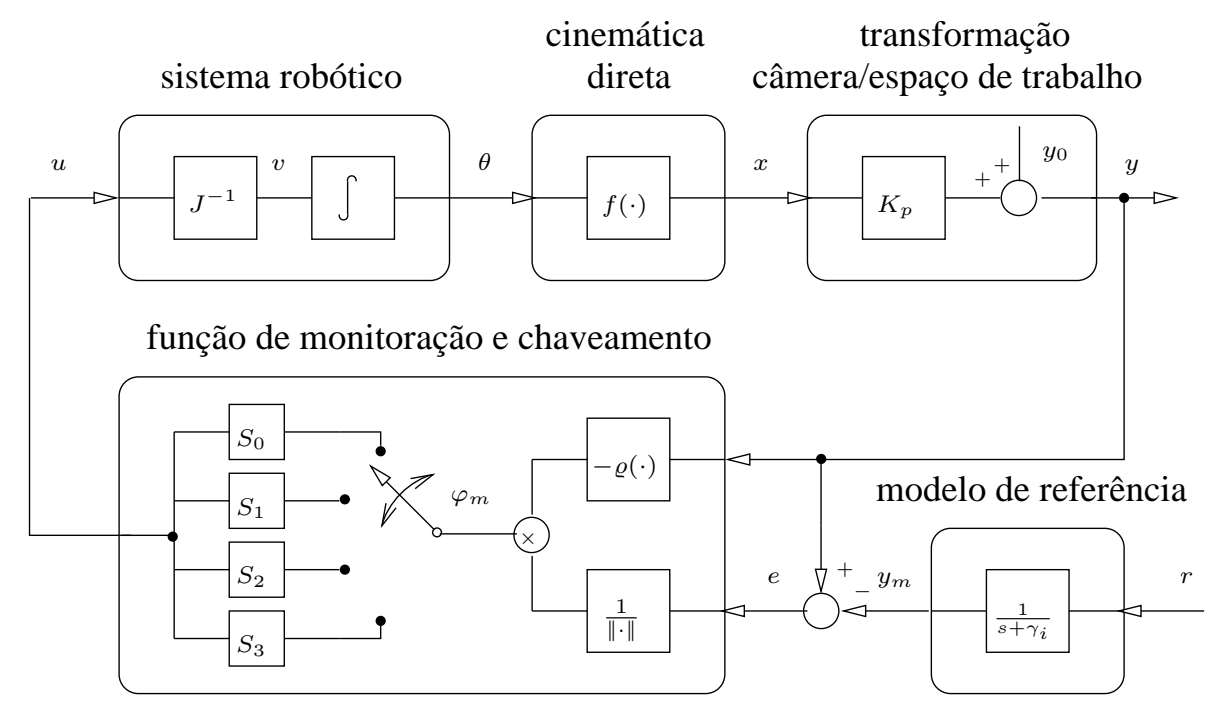

Figura 4: Diagrama em blocos: esquema de controle servovisual proposto.

O processamento de imagem no formato RGB (redgreen-blue) é realizado em uma subjanela de tamanho $100 \times 100$ [pixel]. A primeira estimativa das coordenadas do centróide são executadas off-line utilizando-se uma Interface Gráfica de Usuário (GUI) desenvolvida em linguagem Tcl/Tk (Leite \& Lizarralde, 2006) como mostrado na Figura 5. Durante a execução da tarefa, a característica da imagem é computada utilizando o algoritmo de momentos de imagens (Haralick \& Shapiro, 1993). Devido a sensibilidade ao ruído, o ganho proporcional na malha de controle de velocidade não é grande o suficiente para eliminar o erro em regime permanente devido aos efeitos da gravidade. Essa perturbação foi identificada off-line utilizando um método baseado em mínimos quadrados e depois efetivamente compensada (Spong \& Vidyasagar, 1989).

\subsection{Resultados Experimentais}

Os testes experimentais são realizados sem qualquer procedimento de calibração. A trajetória desejada $y_{m}$ é gerada pelo modelo (4), com $\gamma_{1}=\gamma_{2}=1$, e $r^{T}=\left[r_{1}, r_{2}\right]$ com sinais de referência

$$
\begin{aligned}
& r_{1}=y_{1}(0)+c_{a} R\left[1-\cos \left(\omega_{r} t\right)\right], \\
& r_{2}=y_{2}(0)+c_{b} R\left[\operatorname{sen}\left(\omega_{r} t\right)\right],
\end{aligned}
$$

onde $y^{T}(0)=\left[y_{1}(0), y_{2}(0)\right]$ é a posição inicial das coordenadas do centróide no sistema de coordenadas da imagem, $c_{a}$ e $c_{b}$ são parâmetros constantes que determinam a direção de movimento, $R$ e $\omega_{r}$ são o raio e a velocidade angular da trajetória de referência, respectivamente.

Nesses experimentos, o robô manipulador tem que exe- cutar o rastreamento de uma trajetória circular especificada no sistema de coordenadas da imagem com $R=40$ [pixel] e $\omega_{r}=\frac{\pi}{5}[\mathrm{rad} / \mathrm{s}]$. Outros parâmetros relevantes são: $y_{1}(0)=330$ [pixel], $y_{2}(0)=275$ [pixel] e $c_{a}=c_{b}=1$. $\mathrm{O}$ ângulo inicial de rotação da câmera considerado foi $\psi \approx \pi[\mathrm{rad}]$.

A função de monitoração $\varphi_{m}$ é obtida a partir de (18)(19) com $a(k)=k+1, \lambda_{m}=\lambda_{c}=0.9$. Uma constante de valor 15 foi adicionada a $\varphi_{m}$ para reduzir modificações espúrias na direção de controle devido a ruídos de medição. Além disso, sabe-se que o ruído de medição causa chattering no sinal de controle. Entretanto, este pode ser suavizado utilizando-se o método da camada de fronteira (Edwards \& Spurgeon, 1998) na lei de controle UVC. A função de modulação foi implementada a fim de satisfazer (15) e um limitante superior para $d(\theta)$ foi obtido utilizando-se o mapeamento cinemático direto. Todos os testes foram pensados de forma a evitar singularidades no Jacobiano em (24). Com o intuito de simplificar a implementação do controlador, a função de modulação foi escolhida constante $(\varrho=28)$, mostrando-se suficiente para garantir o rastreamento desejado.

A Figura 6 mostra o comportamento da função de monitoração $\varphi_{m}$ e da norma do erro $|e|$. O sinal de controle foi inicializado com a matriz $S_{0}$ (que não é a matriz correta para $\psi=\pi$ ). Em seguida, o ângulo de orientação da câmera foi modificado para $\psi \approx \pi / 2$. Note que, apenas no quarto chaveamento (SW) pelo conjunto indexado $\mathcal{Q}$, isto é $k=4$, a matriz correta $S_{0}$ é novamente selecionada (considerando agora que $\psi=\pi / 2$ e $-K_{p} S_{0}$ é Hurwitz) e depois disso $|e| \rightarrow 0$. 


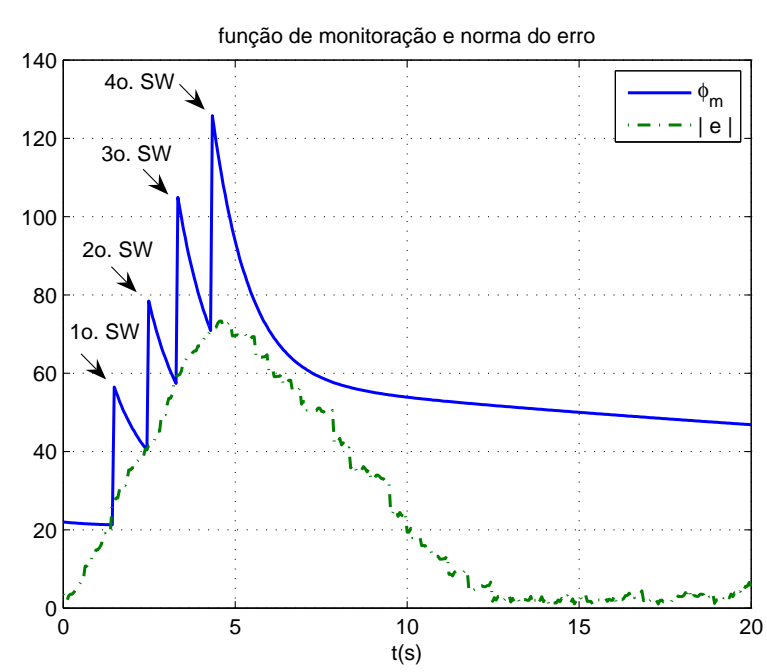

Figura 6: Função de Monitoração $\left(\varphi_{m}\right)$ e norma do erro $(|e|)$.

A Figura 7 descreve o erro de imagem $e(t)$ e o sinal de controle $u(t)$, respectivamente. Note que, a convergência assintótica do erro para um conjunto residual é evidente. A trajetória do alvo é ilustrada na Figura 8, onde observa-se que o rastreamento é alcançado mesmo para $|\psi|>\pi / 2$ e mudanças em $\psi$ artificialmente introduzidas durante o experimento.
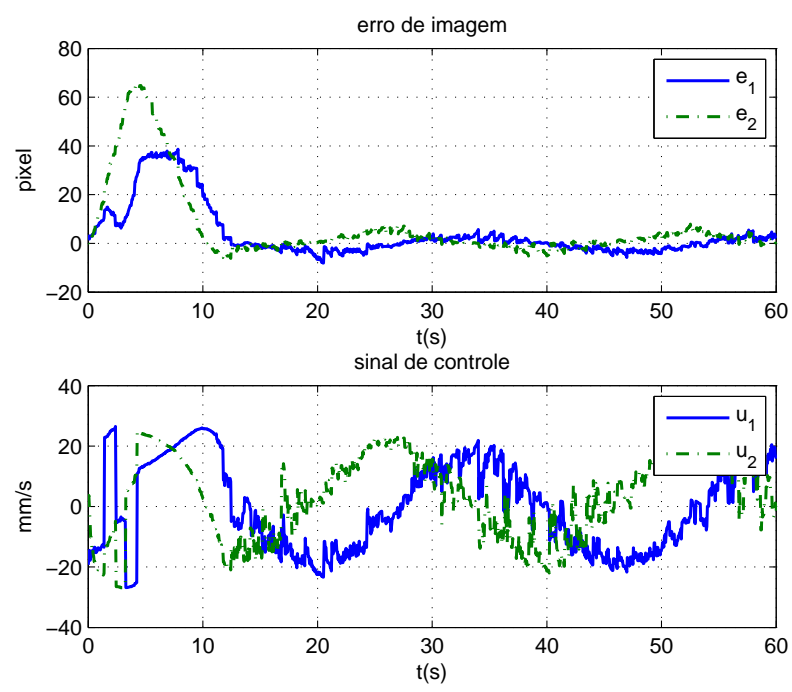

Figura 7: Erro de imagem $(e)$ e sinal de controle $(u)$.

Apesar das incertezas cinemáticas devido a flexibilidade do manipulador robótico e as não-linearidades do tipo backlash provenientes de folgas nas engrenagens das juntas do robô, o controlador vetorial unitário e por mo- dos deslizantes proposto mostrou-se bastante robusto e conseguiu garantir um excelente desempenho no rastreamento de trajetória.

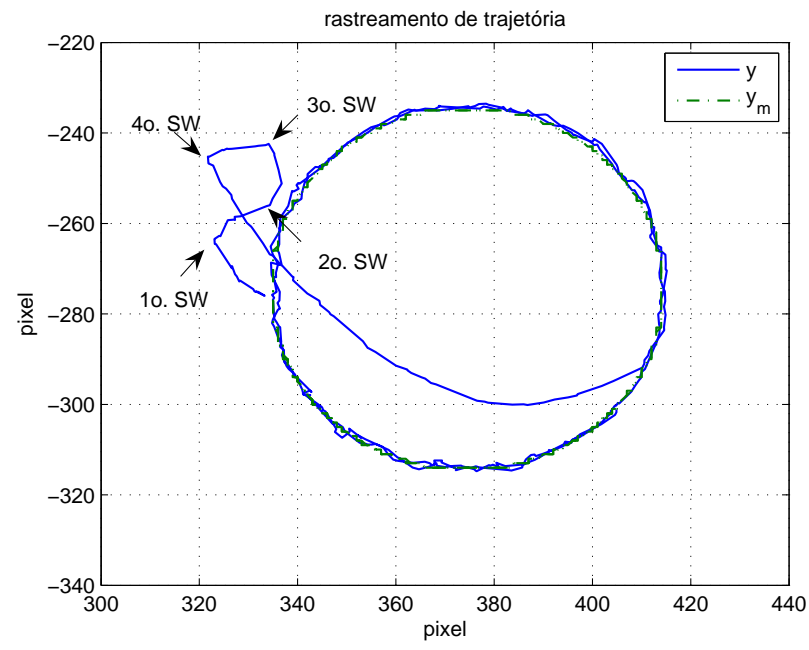

Figura 8: Rastreamento de trajetória no plano da imagem.

\section{CONCLUSÕES}

Um controlador por modos deslizantes utilizando funções de monitoração foi recentemente introduzido para sistemas não-lineares SISO com direção de controle desconhecida. Neste artigo, a abordagem proposta permite incluir não-linearidades fortes no estado não-medido e lidar com sistemas multivariáveis e de grau relativo um. A estratégia é baseada no controle vetorial unitário por realimentação de saída para gerar o modo deslizante ideal e em um esquema de monitoração para reduzir o conhecimento sobre a matriz de ganho de alta frequência usualmente requerido por outros métodos. A estabilidade global assintótica com respeito a um conjunto compacto e o rastreamento exato da saída são provados. O controlador proposto foi testado com sucesso em um experimento de servovisão robótica. Além disso, foi removida a restrição sobre o ângulo de orientação da câmera usualmente requerido nas estratégias adaptativas.

\section{Apêndice}

\section{A DEMONSTRAÇÃO DO TEOREMA 1}

Daqui em diante, $k_{i}>0$ denota constantes independentes das condições iniciais e $\Psi_{i}(\cdot) \in \mathcal{K}$. A prova é conduzida em quatro partes a seguir. 
(1) O chaveamento da direção de controle termina: Motivado pela Observação 1, suponha por contradição que as trocas ou chaveamentos de $S_{q}$ em (17) ocorram sem parar $\forall t \in\left[0, t_{M}\right)$, onde $t_{M}$ pode ser finito ou infinito. Então, $a(k)$ em (18)-(19) cresce ilimitadamente à medida que $k \rightarrow+\infty$. Assim sendo, existe um valor finito $\kappa$ tal que para $k \geq \kappa$ : (i) o termo $a(k) e^{-\lambda_{c} t}$ será um majorante de $\left|\pi_{2}(t)\right|$ em (16) e (ii) $-K_{p} S_{q}$ é Hurwitz. A partir de (i), $\varphi_{m}(t)>\zeta(t), \forall t \in\left[t_{\kappa}, t_{\kappa+1}\right)$, $\operatorname{com} \zeta$ em (16). A partir de (ii), $\zeta$ é um limitante válido para $|e|$. Consequentemente, nenhum chaveamento ocorrerá após $t=t_{\kappa}$, i.e., $t_{\kappa+1}=t_{M}$ (veja (20)), levando-nos assim a uma contradição. Deste modo, o chaveamento de $\varphi_{m}$ (19) tem que parar após um número finito de chaveamentos $k=N$ e $t_{N} \in\left[0, t_{M}\right)$.

(2) Estabilidade com respeito a um conjunto compacto: Não é difícil concluir que $N$ pode ser relacionado com $|z(0)|$, visto que $\pi_{2}(0) \leq k_{1}|z(0)|$ por definição. De fato, pode-se escrever $N \leq \Psi_{1}(|z(0)|)+k_{2}$. Assim, tem-se que $a(N) \leq \Psi_{2}(|z(0)|)+k_{3}$. Agora, a partir de (18)-(21), $|e(t)| \leq\left|e\left(t_{k}\right)\right|+a(k), \forall t \in\left[t_{k}, t_{k+1}\right)$ e a simples desigualdade linear recursiva $\left|e\left(t_{k+1}\right)\right| \leq \varphi_{k} \leq$ $\left|e\left(t_{k}\right)\right|+a(k)$ pode ser obtida, levando à conclusão que $\left|e\left(t_{k}\right)\right| \leq|e(0)|+N a(N)$. Consequentemente, obtém-se $|z(t)| \leq \Psi_{3}(|z(0)|)+c_{z}, \forall t \in\left[0, t_{M}\right)$, onde $c_{z}$ é uma constante positiva. Deste modo, dado $R>c_{z}$, para $|z(0)|<R_{0}$ com $R_{0} \leq \Psi_{3}^{-1}\left(R-c_{z}\right)$, tem-se que $|z(t)|$ estará sempre limitado por $R$ à medida que $t \rightarrow t_{M}$. Isso implica que $z(t)$ é uniformemente limitado e não pode escapar em tempo finito, isto é, $t_{M}=+\infty$. Por esta razão, a estabilidade com respeito à bola de raio $c_{z}$ é garantida para $z(0)$ dentro da bola- $R_{0}$. Uma vez que $R$ e, portanto, $R_{0}$ podem ser escolhidos arbitrariamente grandes, a estabilidade global é concluída.

(3) Sinais limitados e convergência exponencial para zero: Uma vez que o chaveamento da direção de controle pára e $\varphi_{m}$ converge para zero exponencialmente, então, conclui-se (independentemente de uma matriz $-K_{p} S_{q}$ Hurwitz ser selecionada em $k=N)$ que $e(t)$ e $z(t)$ em (22) convergirão para zero ao menos exponencialmente. Relembrando que $y_{m}$ é uniformemente limitado e que $y=e+y_{m}$, a partir de (H2) conclui-se que todos os sinais do sistema em malha fechada são uniformemente limitados.

(4) Modo Deslizante Ideal: É sabido que se $-K_{p} S_{q}$ é Hurwitz, todas as trajetórias do sistema convergem para a origem do espaço de estado do erro (Cunha et al., 2003, Lemma 1). Além disso, se $-K_{p} S_{q}$ não é Hurwitz, então para quase toda condição inicial (i.e., exceto para um conjunto de medida nula) as trajetórias do sistema divergem ilimitadamente ou não convergem para a origem.
Isso é uma contradição, visto que se o processo de chaveamento cessa, de acordo com o item (3) acima, o estado deve convergir para a origem. Assim, quase sempre, a última matriz $S_{q}$ selecionada é tal que $-K_{p} S_{q}$ seja Hurwitz. Portanto, a partir de (Cunha et al., 2003, Lemma 1), podemos adicionalmente concluir que $e(t)$ torna-se identicamente nulo após um tempo finito desde que $\delta>0$ em (15).

\section{AGRADECIMENTOS}

Este trabalho contou com o apoio financeiro da FAPERJ e do CNPq. Os autores também agradecem a Antonio C. Leite por sua valiosa ajuda na execução dos testes experimentais realizados.

\section{REFERÊNCIAS}

Arcak, M., Larsen, M. \& Kokotović, P. (2002). Boundedness without absolute stability in systems with stiffening nonlinearities, European J. of Control. 8(3): 243-250.

Astolfi, A., Hsu, L., Netto, M. \& Ortega, R. (2002). Two solutions to the adaptive visual servoing problem, IEEE Trans. Robotics and Automation 18(3): 387-392.

Baida, S. V. (1993). Unit sliding mode control in continuous- and discrete-time systems, Int. J. Contr. 57(5): 1125-1132.

Bartolini, G., Ferrara, A. \& Giacomini, L. (2003). A switching controller for systems with hard uncertainties, IEEE Trans. on Circuits and Sytems. 50(8): 984-990.

Bartolini, G., Pisano, A. \& Usai, E. (2009). On the second-order sliding mode control of nonlinear systems with uncertain control direction, Automatica 45: 2982-2985.

Chien, C.-J., Sun, K.-C., Wu, A.-C. \& Fu, L.-C. (1996). A robust MRAC using variable structure design for multivariable plants, Automatica 32(6): 833-848.

Cunha, J. P. V. S. (2004). Controle de Sistemas Multivariáveis Incertos por Realimentação de Saída e Modo Deslizante, $\mathrm{PhD}$ thesis, Ph.D. thesis, Programa de Engenharia Elétrica, COPPE/UFRJ, Rio de Janeiro.

Cunha, J. P. V. S., Hsu, L., Costa, R. R. \& Lizarralde, F. (2003). Output-feedback model-reference sliding mode control of uncertain multivariable systems, IEEE Trans. Aut. Contr. 48(12): 2245-2250.

Deaecto, G. S. \& Geromel, J. C. (2009). Controle de sistemas lineares com comutação, Controle $\mathcal{E}$ Automação 19: 431-443.

Drakunov, S. (1993). Sliding mode control of the systems with uncertain direction of control vector, Proc. IEEE Conf. on Decision and Control, San Antonio, pp. 2477-2478.

Edwards, C. \& Spurgeon, S. K. (1998). Sliding Mode Control: Theory and Applications, Taylor \& Francis Ltd.

Filippov, A. F. (1964). Differential equations with discontinuous right-hand side, American Math. Soc. Translations 42(2): 199-231.

Freidovich, L. B. \& Khalil, H. K. (2007). Lyapunov-based switching control of nonlinear systems using high-gain observers, Automatica (43): 150-157. 
Fridman, L., Shtessel, Y., Edwards, C. \& Yan, X. G. (2008) Higher-order sliding-mode observer for state estimation and input reconstruction in nonlinear systems, Int. J. of Robust and Nonlinear Control 18: 399-412.

Fu, M. \& Barmish, B. (1986). Adaptive stabilization of linear systems via switching control, IEEE Trans. Aut. Contr. 31(12): 1097-1103.

Haralick, R. M. \& Shapiro, L. G. (1993). Computer and Robot Vision, Vol. II, Addison-Wesley Inc.

Hespanha, J. P., Liberzon, D. \& Morse, A. (2003). Overcoming the limitations of adaptive control by means of logic-based switching, Systems \& Contr. Letters 49: 49-65.

Hsu, L., Costa, R. R. \& Cunha, J. P. V. S. (2003). Model-reference output-feedback sliding mode controller for a class of multivariable nonlinear systems, Asian Journal of Control 5(4): 543556.

Hsu, L., Cunha, J. P. V. S., Costa, R. R. \& Lizarralde, F. (2002) Multivariable output-feedback sliding mode control, in X. Yu \& J.-X. Xu (eds), Variable Structure Systems: Towards the 21st Century, Springer-Verlag, pp. 283-313.

Hutchinson, S., Hager, G. \& Corke, P. (1996). A tutorial on visual servo control, IEEE Trans. Robotics and Automation 12(5): 651-670.

Jiang, Z. P., Mareels, I., Hill, D. J. \& Huang, J. (2004). A unifying framework for global regulation nonlinear output feedback: from ISS to iISS, IEEE Trans. Aut. Contr. 49(4): 549-562.

Kelly, R., Reyes, R., Moreno, J. \& Hutchinson, S. (1999). A two loops direct visual control of direct-drive planar robots with moving target, pp. 599-604.

Khalil, H. K. (2002). Nonlinear Systems, $3^{\text {rd }}$ edn, Prentice Hall.

Krichman, M., Sontag, E. D. \& Wang, Y. (2001). Input-outputto-state stability, SIAM J. Contr. Optim. 39(6): 1874-1928.

Leite, A. C. \& Lizarralde, F. (2006). Application of Tcl/Tk for a robotic system, Proceedings of 13 th Annual Tcl/Tk Conference, pp. 1-3.

Leite, A. C., Lizarralde, F., Hsu, L. \& Zachi, A. R. L. (2004). Rastreamento de trajetórias por servovisão adaptativa, Controle ES Automação 15: 309-319.

Martensson, B. (1991). The unmixing problem, IMA J. Math. Contr. Info. 8: 367-377.

Mudgett, D. \& Morse, A. (1985). Adaptive stabilization of linear systems with unknown high frequency gains, IEEE Trans. Aut. Contr. 30: 549-554.

Nunes, E. V. L., Peixoto, A. J., Oliveira, T. R. \& Hsu, L. (2010) Global exact tracking for uncertain multivariable linear systems by output feedback sliding mode control, Proc. American Contr. Conf., Baltimore, pp. 974-979.

Nussbaum, R. (1983). Some results on a conjecture in parameter adaptive control, Systems \& Contr. Letters 3: 243-246.

Oliveira, T. R., Peixoto, A. J. \& Hsu, L. (2010). Controle por realimentação de saída para sistemas incertos fortemente nãolineares, Controle \& Automação 21: 69-81.

Oliveira, T. R., Peixoto, A. J., Nunes, E. V. L. \& Hsu, L. (2007). Control of uncertain nonlinear systems with arbitrary relative degree and unknown control direction using sliding modes, Int. J. Adaptive Control Signal Process. 21: 692-707.

Papanikolopoulos, N. \& Khosla, P. (1994). Adaptive robotic visual tracking: Theory and experiments, IEEE Trans. Aut. Contr. 38(3): 429-445.
Ryan, E. (1993). Adaptive stabilization of multi-input nonlinear systems, Int. J. of Robust and Nonlinear Control 3(2): 169181.

Sontag, E. D. \& Wang, Y. (1997). Output-to-state stability and detectability of nonlinear systems, Systems $\&$ Contr. Letters 29: 279-290.

Spong, M. W. \& Vidyasagar, M. (1989). Robot Dynamics and Control, John Wiley \& Sons.

Tao, G. \& Ioannou, P. A. (1989). A MRAC for multivariable plants with zero residual tracking error, Proc. IEEE Conf. on Decision and Control, Tampa, USA, pp. 1597-1600.

Van der Schaft, A. \& Schumacher, H. (2000). An Introduction to Hybrid Dynamical Systems, Springer, London.

Wu, Y. \& Zhou, Y. (2004). Output feedback control for MIMO non-linear systems with unknown sign of the high frequency gain matrix, Int. J. Contr. 77(1): 9-18.

Yan, L., Hsu, L., Costa, R. R. \& Lizarralde, F. (2008). A variable structure model reference robust control without a prior knowledge of high frequency gain sign, Automatica 44: 10361044 .

Zachi, A. R. L., Hsu, L., Ortega, R. \& Lizarralde, F. (2006). Dynamic control of uncertain manipulators through immersion and invariance adaptive visual servoing, The International Journal of Robotics Research 25(11): 1149-1159.

Zachi, A. R. L., Lizarralde, F., Hsu, L. \& Leite, A. C. (2006). Adaptive control of nonlinear visual servoing systems for $3 \mathrm{D}$ cartesian tracking, Controle \& A Automação 17: 381-390.

Zergeroglu, E., Dawson, D. M., de Queiroz, M. S. \& Nagarkatti, S. (1999). Robust visual-servo control of robot manipulators in the presence of uncertainty, Proc. IEEE Conf. on Decision and Control pp. 4137-4142.

Zhang, T. P. \& Ge, S. S. (2007). Adaptive neural control of MIMO nonlinear state time-varying delay systems with unknown dead-zones and gain signs, Automatica 43: 1021-1033. 